\title{
Boundary value problems for impulsive multi-order Hadamard fractional differential equations
}

\author{
Weera Yukunthorn', Suthep Suantai ${ }^{2}$, Sotiris K Ntouyas ${ }^{3,4}$ and Jessada Tariboon ${ }^{1 *}$
}

\author{
"Correspondence: \\ jessada.t@sci.kmutnb.ac.th \\ ${ }^{1}$ Nonlinear Dynamic Analysis \\ Research Center, Department of \\ Mathematics, Faculty of Applied \\ Science, King Mongkut's University \\ of Technology North Bangkok, \\ Bangkok, 10800, Thailand \\ Full list of author information is \\ available at the end of the article
}

\begin{abstract}
In this paper, we study the existence and uniqueness of solutions for impulsive multi-orders Caputo-Hadamard fractional differential equations equipped with boundary and integral conditions. The Banach, Schaefer, and Rothe fixed point theorems and degree theory are used to establish our main results. Examples illustrating the main results are presented.
\end{abstract}

MSC: 34A08; 34B10; 34A37

Keywords: impulsive differential equations; Hadamard fractional calculus; fixed point theorems; existence

\section{Introduction}

During the last years, fractional calculus has gained considerable importance due to the applications in almost all applied sciences. It was pointed out that fractional derivatives and integrals are more convenient for describing real materials, and some physical problems were treated by using derivatives of non-integer orders. For details, and some recent results on the subject we refer to [1-9] and the references cited therein.

It has been noticed that most of the work on the topic is based on Riemann-Liouville and Caputo type fractional differential equations. Another kind of fractional derivatives that appears side by side to Riemann-Liouville and Caputo derivatives in the literature is the fractional derivative due to Hadamard introduced in 1892 [10], which differs from the preceding ones in the sense that the kernel of the integral (in the definition of Hadamard derivative) contains a logarithmic function of arbitrary exponent. Details and properties of the Hadamard fractional derivative and integral can be found in [1,11-15]. However, this calculus with Hadamard derivatives is still studied less than that of Riemann-Liouville.

On the other hand, integer order impulsive differential equations have become important in recent years as mathematical models of phenomena in both the physical and the social sciences. There has a significant development in impulsive theory especially in the area of impulsive differential equations with fixed moments; see for instance [16-20].

Recently in [21], Wang et al. studied existence and uniqueness results for the following impulsive multipoint fractional integral boundary value problem involving multi-order

(c) 2015 Yukunthorn et al. This article is distributed under the terms of the Creative Commons Attribution 4.0 International License (http://creativecommons.org/licenses/by/4.0/), which permits unrestricted use, distribution, and reproduction in any medium, provided you give appropriate credit to the original author(s) and the source, provide a link to the Creative Commons license, and indicate if changes were made. 
fractional derivatives and a deviating argument:

$$
\left\{\begin{array}{l}
{ }^{c} D_{t_{k}^{+}}^{\alpha_{k}} u(t)=f(t, u(t), u(\theta(t))), \quad 1<\alpha_{k} \leq 2, \\
\Delta u\left(t_{k}\right)=I_{k}\left(u\left(t_{k}\right)\right), \quad \Delta u^{\prime}\left(t_{k}\right)=I_{k}^{*}\left(u\left(t_{k}\right)\right), \quad k=1,2, \ldots, p, \\
u(0)=\sum_{k=0}^{p} \lambda_{k} \mathcal{J}_{t_{k}^{+}}^{\beta_{k}} u\left(\eta_{k}\right), \quad u^{\prime}(0)=0, \quad t_{k}<\eta_{k}<t_{k+1},
\end{array}\right.
$$

where ${ }^{c} D_{t_{k}^{+}}^{\alpha_{k}}$ is the Caputo fractional derivative of order $\alpha_{k}, \mathcal{J}_{t_{k}^{+}}^{\beta_{k}}$ is Riemann-Liouville fractional integral of order $\beta_{k}>0, f \in C(J \times \mathbb{R} \times \mathbb{R}, \mathbb{R}), I_{k}, I_{k}^{*} \in C(\mathbb{R}, \mathbb{R}), \theta \in C(J, J), J=[0, T]$ $(T>0), 0=t_{0}<t_{1}<\cdots<t_{k}<\cdots<t_{p}<t_{p+1}=T, \Delta u\left(t_{k}\right)=u\left(t_{k}^{+}\right)-u\left(t_{k}^{-}\right)$, and $\Delta u^{\prime}\left(t_{k}\right)=$ $u^{\prime}\left(t_{k}^{+}\right)-u^{\prime}\left(t_{k}^{-}\right)$where $u\left(t_{k}^{+}\right), u^{\prime}\left(t_{k}^{+}\right)$and $u\left(t_{k}^{-}\right), u^{\prime}\left(t_{k}^{-}\right)$denote the right and left hand limits of $u(t)$ and $u^{\prime}(t)$ at $t=t_{k}(k=1,2, \ldots, p)$.

In 2015, Wang et al. [22] established the existence of solutions for a class of nonlinear impulsive Hadamard fractional differential equations with initial condition of the form

$$
\left\{\begin{array}{l}
{ }_{H} D_{1^{+}}^{\alpha} u(t)=f(t, u(t)), \quad \alpha \in(0,1), t \in(1, e] \backslash\left\{t_{1}, t_{2}, \ldots, t_{m}\right\}, \\
\Delta u\left(t_{i}\right)={ }_{H} J_{1^{+}}^{1-\alpha} u\left(t_{i}^{+}\right)-{ }_{H} J_{1^{+}}^{1-\alpha} u\left(t_{i}^{-}\right)=p_{i}, \quad p_{i} \in \mathbb{R}, i=1,2, \ldots, m, \\
{ }_{H} J_{1^{+}}^{1-\alpha} u\left(1^{+}\right)=u_{0}, \quad u_{0} \in \mathbb{R},
\end{array}\right.
$$

where ${ }_{H} D_{1^{+}}^{\alpha}$ is the left-side Hadamard fractional derivative of order $\alpha$ with the lower limit 1 and $H_{1^{+}}^{1-\alpha}$ denotes left-side Hadamard fractional integral of order $1-\alpha$. The existence results were obtained by using the Banach contraction principle and Schauder's fixed point theorem on the weight spaces of piecewise continuous functions.

The Hadamard and Riemann-Liouville fractional derivatives have one similar property, which is the fact that the derivative of a constant is not equal to zero. It is caused by the definitions of them containing the usual derivative outside the integrals. In 2012, Jarad et al. [23] presented the modifications of the Hadamard fractional derivative into a more suitable one having physically interpretable initial conditions similar to the Caputo sense. In 2014, Gambo et al. [24] proved the fundamental theorem of fractional calculus, some interesting results and also semigroup properties of Caputo-Hadamard operators.

In this paper we are concerned with the existence of solutions for boundary value problems of impulsive Hadamard fractional differential equations of the form

$$
\left\{\begin{array}{l}
{ }^{C} \mathcal{D}_{t_{k}}^{p_{k}} x(t)=f(t, x(t)), \quad t \in J_{k} \subset\left[t_{0}, T\right], t \neq t_{k} \\
\Delta x\left(t_{k}\right)=\varphi_{k}\left(x\left(t_{k}\right)\right), \quad k=1,2, \ldots, m \\
\alpha x\left(t_{0}\right)+\beta x(T)=\sum_{i=0}^{m} \gamma_{i} \mathcal{J}_{t_{i}}^{q_{i}} x\left(t_{i+1}\right)
\end{array}\right.
$$

where ${ }^{C} \mathcal{D}_{t_{k}}^{p_{k}}$ is the Hadamard fractional derivative of Caputo type of order $0<p_{k} \leq 1$ on intervals $J_{k}:=\left(t_{k}, t_{k+1}\right], k=1,2, \ldots, m$, with $J_{0}=\left[t_{0}, t_{1}\right], 0<t_{0}<t_{1}<t_{2}<\cdots<t_{k}<\cdots<t_{m}<$ $t_{m+1}=T$ are the impulse points, $J:=\left[t_{0}, T\right], f: J \times \mathbb{R} \rightarrow \mathbb{R}$ is a continuous function, $\varphi_{k} \in$ $C(\mathbb{R}, \mathbb{R}), \mathcal{J}_{t_{i}}^{q_{i}}$ is the Hadamard fractional integral of order $q_{i}>0, i=0,1, \ldots, m$. The jump conditions are defined by $\Delta x\left(t_{k}\right)=x\left(t_{k}^{+}\right)-x\left(t_{k}\right), x\left(t_{k}^{+}\right)=\lim _{\varepsilon \rightarrow 0^{+}} x\left(t_{k}+\varepsilon\right), k=1,2,3, \ldots, m$. 
The paper is organized as follows: Section 2 contains some preliminary notations, definitions and lemmas that we need in the sequel. In Section 3 we present the main results for the problem (1.3), where existence and uniqueness results are proved by using Banach and Rothe fixed point theorems, Leray-Schauder alternative and degree theory. Examples illustrating the obtained results are also presented.

\section{Preliminaries}

In this section, we introduce some notations and definitions of Hadamard fractional calculus (see [1]) and present preliminary results needed in our proofs later.

Definition 2.1 For an at least $n$-times differentiable function $g:[a, b] \rightarrow \mathbb{R}, a, b>0$, the Caputo type Hadamard derivative of fractional order $\alpha$ is defined as

$$
{ }^{C} \mathcal{D}_{a}^{\alpha} g(t)=\frac{1}{\Gamma(n-\alpha)} \int_{a}^{t}\left(\log \frac{t}{s}\right)^{n-\alpha-1} \delta^{n} g(s) \frac{d s}{s}, \quad n-1<\alpha<n, n=[\alpha]+1,
$$

where $\delta=t \frac{d}{d t}, t \in[a, b]$, and $[\alpha]$ denotes the integer part of the real number $\alpha$ and $\log (\cdot)=$ $\log _{e}(\cdot)$.

Definition 2.2 The Hadamard fractional integral of order $\alpha$ is defined as

$$
\mathcal{J}_{a}^{\alpha} g(t)=\frac{1}{\Gamma(\alpha)} \int_{a}^{t}\left(\log \frac{t}{s}\right)^{\alpha-1} g(s) \frac{d s}{s}, \quad \alpha>0,
$$

provided the integral exists on $[a, b]$.

Lemma 2.1 [23] Let $x \in A C_{\delta}^{n}[a, b]$ or $C_{\delta}^{n}[a, b]$ and $\alpha \in \mathbb{C}$, where $X_{\delta}^{n}[a, b]=\{g:[a, b] \rightarrow \mathbb{C}$ : $\left.\delta^{n-1} g(t) \in X[a, b]\right\}$. Then we have

$$
\mathcal{J}_{a}^{\alpha}\left({ }^{C} \mathcal{D}_{a}^{\alpha}\right) x(t)=x(t)-\sum_{k=0}^{n-1} \frac{\delta^{k} x(a)}{k !} \log \left(\frac{t}{a}\right)^{k} .
$$

The key tools for proving of our results are based on the following fixed point theorems.

Theorem 2.1 [25] Suppose that $A: \bar{\Omega} \rightarrow E$ is a completely continuous operator. If one of the following conditions is satisfied:

(i) (Altman) $\|A x-x\|^{2} \geq\|A x\|^{2}-\|x\|^{2}$, for all $x \in \partial \Omega$,

(ii) (Rothe) $\|A x\| \leq\|x\|$, for all $x \in \partial \Omega$,

(iii) (Petryshyn) $\|A x\| \leq\|A x-x\|$, for all $x \in \partial \Omega$,

then $\operatorname{deg}(I-A, \Omega, \theta)=1$, and hence $A$ has at least one fixed point in $\Omega$.

Theorem 2.2 [25] Suppose that $A: \bar{\Omega} \rightarrow$ E is completely continuous operator. If

$$
A x \neq \lambda x, \quad \forall x \in \partial \Omega, \lambda \geq 1,
$$

then $\operatorname{deg}(I-A, \Omega, \theta)=1$ and $A$ has at least one fixed point in $\bar{\Omega}$. 
Theorem 2.3 [26] Let E be a Banach space. Assume that $T: E \rightarrow E$ is a completely continuous operator and the set

$$
V=\{u \in E: u=\lambda T u, 0<\lambda<1\}
$$

is bounded. Then T has a fixed point in $E$.

Lemma 2.2 Assume that $\Phi=\alpha+\beta-\sum_{i=1}^{m} \frac{\gamma_{i}\left(\log \left(t_{i+1} / t_{i}\right) q_{i}\right.}{\Gamma\left(q_{i}+1\right)} \neq 0$. Then the solution of the problem (1.3) is equivalent to the following integral equation:

$$
\begin{aligned}
x(t)= & \mathcal{J}_{t_{k}}^{p_{k}} f(t, x(t))+\sum_{i=0}^{k-1}\left(\mathcal{J}_{t_{i}}^{p_{i}} f\left(t_{i+1}, x\left(t_{i+1}\right)\right)+\varphi_{i+1}\left(x\left(t_{i+1}\right)\right)\right) \\
& +\frac{1}{\Phi}\left[\sum_{i=0}^{m} \gamma_{i} \mathcal{J}_{t_{i}}^{q_{i}+p_{i}} f\left(t_{i+1}, x\left(t_{i+1}\right)\right)-\beta \mathcal{J}_{t_{m}}^{p_{m}} f(T, x(T))\right. \\
& -\beta \sum_{i=0}^{m-1}\left(\mathcal{J}_{t_{i}}^{p_{i}} f\left(t_{i+1}, x\left(t_{i+1}\right)\right)+\varphi_{i+1}\left(x\left(t_{i+1}\right)\right)\right) \\
& \left.+\sum_{i=1}^{m}\left(\frac{\gamma_{i}\left(\log \left(t_{i+1} / t_{i}\right)\right)^{q_{i}}}{\Gamma\left(q_{i}+1\right)}\right)\left(\sum_{j=0}^{i-1}\left(\mathcal{J}_{t_{j}}^{p_{j}} f\left(t_{j+1}, x\left(t_{j+1}\right)\right)+\varphi_{j+1}\left(x\left(t_{j+1}\right)\right)\right)\right)\right] .
\end{aligned}
$$

Proof By Lemma 2.1, the solution of (1.3) on interval $J_{0}$ can be written as

$$
x(t)=\mathcal{J}_{t_{0}}^{p_{0}} f(t, x(t))+x_{0},
$$

where $x_{0} \in \mathbb{R}$. For $t \in J_{1}$, by using Lemma 2.1 and the impulse condition $\Delta x\left(t_{1}\right)=\varphi_{1}\left(x\left(t_{1}\right)\right)$, we obtain

$$
\begin{aligned}
x(t) & =\mathcal{J}_{t_{1}}^{p_{1}} f(t, x(t))+x\left(t_{1}^{+}\right) \\
& =\mathcal{J}_{t_{1}}^{p_{1}} f(t, x(t))+\mathcal{J}_{t_{0}}^{p_{0}} f\left(t_{1}, x\left(t_{1}\right)\right)+\varphi_{1}\left(x\left(t_{1}\right)\right)+x_{0} .
\end{aligned}
$$

Again, for $t \in J_{2}$, we have

$$
\begin{aligned}
x(t) & =\mathcal{J}_{t_{2}}^{p_{2}} f(t, x(t))+x\left(t_{2}^{+}\right) \\
& =\mathcal{J}_{t_{2}}^{p_{2}} f(t, x(t))+\mathcal{J}_{t_{1}}^{p_{1}} f\left(t_{2}, x\left(t_{2}\right)\right)+\varphi_{2}\left(x\left(t_{2}\right)\right)+\mathcal{J}_{t_{0}}^{p_{0}} f\left(t_{1}, x\left(t_{1}\right)\right)+\varphi_{1}\left(x\left(t_{1}\right)\right)+x_{0} .
\end{aligned}
$$

Repeating the above process, for $t \in J$, we obtain

$$
x(t)=\mathcal{J}_{t_{k}}^{p_{k}} f(t, x(t))+\sum_{i=0}^{k-1}\left(\mathcal{J}_{t_{i}}^{p_{i}} f\left(t_{i+1}, x\left(t_{i+1}\right)\right)+\varphi_{i+1}\left(x\left(t_{i+1}\right)\right)\right)+x_{0} .
$$

Applying the boundary condition of (1.3), it follows that

$$
\begin{aligned}
\alpha x\left(t_{0}\right)+\beta x(T)= & (\alpha+\beta) x_{0}+\beta \mathcal{J}_{t_{m}}^{p_{m}} f(T, x(T)) \\
& +\beta \sum_{i=0}^{m-1}\left(\mathcal{J}_{t_{i}}^{p_{i}} f\left(t_{i+1}, x\left(t_{i+1}\right)\right)+\varphi_{i+1}\left(x\left(t_{i+1}\right)\right)\right)
\end{aligned}
$$


and

$$
\begin{aligned}
& \sum_{i=0}^{m} \gamma_{i} \mathcal{J}_{t_{i}}^{q_{i}} x\left(t_{i+1}\right) \\
& =\sum_{i=0}^{m} \gamma_{i} \mathcal{J}_{t_{i}}^{p_{i}+q_{i}} f\left(t_{i+1}, x\left(t_{i+1}\right)\right)+x_{0} \sum_{i=0}^{m} \frac{\gamma_{i}\left(\log \left(t_{i+1} / t_{i}\right)\right)^{q_{i}}}{\Gamma\left(q_{i}+1\right)} \\
& \quad+\sum_{i=1}^{m}\left(\frac{\gamma_{i}\left(\log \left(t_{i+1} / t_{i}\right)\right)^{q_{i}}}{\Gamma\left(q_{i}+1\right)}\right)\left(\sum_{j=0}^{i-1}\left(\mathcal{J}_{t_{j}}^{j-1} f\left(t_{j+1}, x\left(t_{j+1}\right)\right)+\varphi_{j+1}\left(x\left(t_{j+1}\right)\right)\right)\right)
\end{aligned}
$$

which leads to

$$
\begin{aligned}
x_{0}= & \frac{1}{\Phi}\left[\sum_{i=0}^{m} \gamma_{i} \mathcal{J}_{t_{i}}^{q_{i}+p_{i}} f\left(t_{i+1}, x\left(t_{i+1}\right)\right)-\beta \mathcal{J}_{t_{m}}^{p_{m}} f(T, x(T))\right. \\
& -\beta \sum_{i=0}^{m-1}\left(\mathcal{J}_{t_{i}}^{p_{i}} f\left(t_{i+1}, x\left(t_{i+1}\right)\right)+\varphi_{i+1}\left(x\left(t_{i+1}\right)\right)\right) \\
& \left.+\sum_{i=1}^{m}\left(\frac{\gamma_{i}\left(\log \left(t_{i+1} / t_{i}\right)\right)^{q_{i}}}{\Gamma\left(q_{i}+1\right)}\right)\left(\sum_{j=0}^{i-1}\left(\mathcal{J}_{t_{j}}^{j-1} f\left(t_{j+1}, x\left(t_{j+1}\right)\right)+\varphi_{j+1}\left(x\left(t_{j+1}\right)\right)\right)\right)\right] .
\end{aligned}
$$

Replacing the constant $x_{0}$ into (2.2), we obtain (2.1), as desired.

\section{Main results}

Let $P C(J, \mathbb{R})=\left\{x: J \rightarrow \mathbb{R} ; x(t)\right.$ is continuous everywhere except for some $t_{k}$ at which $x\left(t_{k}^{+}\right)$ and $x\left(t_{k}^{-}\right)$exist and $\left.x\left(t_{k}^{-}\right)=x\left(t_{k}\right), k=1,2, \ldots, m\right\}$. Obviously, $P C(J, \mathbb{R})$ is a Banach space with the norm $\|x\|=\sup \{|x(t)| ; t \in J\}$. A function $x \in P C$ is called a solution of the problem (1.3) if it satisfies (1.3).

In this section, we investigate the existence and uniqueness of solutions for the problem (1.3) via a variety of fixed point theorems by defining an operator $\mathcal{K}: P C \rightarrow P C$ as

$$
\begin{aligned}
\mathcal{K} x(t)= & \mathcal{J}_{t_{k}}^{p_{k}} f(t, x(t))+\sum_{i=0}^{k-1}\left(\mathcal{J}_{t_{i}}^{p_{i}} f\left(t_{i+1}, x\left(t_{i+1}\right)\right)+\varphi_{i+1}\left(x\left(t_{i+1}\right)\right)\right) \\
& +\frac{1}{\Phi}\left[\sum_{i=0}^{m} \gamma_{i} \mathcal{J}_{t_{i}}^{q_{i}+p_{i}} f\left(t_{i+1}, x\left(t_{i+1}\right)\right)-\beta \mathcal{J}_{t_{m}}^{p_{m}} f(T, x(T))\right. \\
& -\beta \sum_{i=0}^{m-1}\left(\mathcal{J}_{t_{i}}^{p_{i}} f\left(t_{i+1}, x\left(t_{i+1}\right)\right)+\varphi_{i+1}\left(x\left(t_{i+1}\right)\right)\right) \\
& \left.+\sum_{i=1}^{m}\left(\frac{\gamma_{i}\left(\log \left(t_{i+1} / t_{i}\right)^{q_{i}}\right)}{\Gamma\left(q_{i}+1\right)}\right)\left(\sum_{j=0}^{i-1}\left(\mathcal{J}_{t_{j}}^{p_{j}} f\left(t_{j+1}, x\left(t_{j+1}\right)\right)+\varphi_{j+1}\left(x\left(t_{j+1}\right)\right)\right)\right)\right] .
\end{aligned}
$$

Clearly, the boundary value problem (1.3) becomes a fixed point problem $x=\mathcal{K} x$. 
For convenience, we set the notations of constants, thus

$$
\begin{aligned}
\Lambda_{1}= & \frac{\left(\log \left(t / t_{k}\right)\right)^{p_{k}}}{\Gamma\left(p_{k}+1\right)}+\sum_{i=0}^{m-1} \frac{\left(\log \left(t_{i+1} / t_{i}\right)\right)^{p_{i}}}{\Gamma\left(p_{i}+1\right)} \\
& +\frac{1}{|\Phi|}\left\{\sum_{i=0}^{m} \frac{\left|\gamma_{i}\right|\left(\log \left(t_{i+1} / t_{i}\right)\right)^{q_{i}+p_{i}}}{\Gamma\left(q_{i}+p_{i}+1\right)}+|\beta| \sum_{i=0}^{m} \frac{\left(\log \left(t_{i+1} / t_{i}\right)\right)^{p_{i}}}{\Gamma\left(p_{i}+1\right)}\right. \\
& \left.+|\beta| \frac{\left(\log \left(T / t_{m}\right)\right)^{p_{m}}}{\Gamma\left(p_{m}+1\right)}+\sum_{i=1}^{m} \sum_{j=0}^{i-1}\left(\frac{\left(\log \left(t_{j+1} / t_{j}\right)\right)^{p_{j}}}{\Gamma\left(p_{j}+1\right)}\right)\left(\frac{\left|\gamma_{i}\right|\left(\log \left(t_{i+1} / t_{i}\right)\right)^{q_{i}}}{\Gamma\left(q_{i}+1\right)}\right)\right\}, \\
\Lambda_{2}= & k+\frac{\left(\log \left(t / t_{k}\right)\right)^{p_{k}}}{\Gamma\left(p_{k}+1\right)}+\frac{1}{\Phi}\left[|\beta| m+\sum_{i=0}^{m} \frac{\left|\gamma_{i}\right|\left(\log \left(t_{i+1} / t_{i}\right)\right)^{q_{i}}}{\Gamma\left(q_{i}+1\right)}+|\beta| \frac{\left(\log \left(T / t_{m}\right)\right)^{p_{m}}}{\Gamma\left(p_{m}+1\right)}\right] .
\end{aligned}
$$

Theorem 3.1 Assume that $f: J \times \mathbb{R} \rightarrow \mathbb{R}$ and $\varphi_{k}: \mathbb{R} \rightarrow \mathbb{R}, k=1,2, \ldots, m$, are continuous functions which satisfy the following conditions:

$\left(\mathrm{H}_{1}\right) \quad|f(t, x)-f(t, y)| \leq L_{1}|x-y|, \forall t \in J, L_{1}>0, x, y \in \mathbb{R}$

$\left(\mathrm{H}_{2}\right)\left|\varphi_{k}(u)-\varphi_{k}(v)\right| \leq L_{2}|u-v|, L_{2}>0$, for all $u, v \in \mathbb{R}, \forall k=1,2, \ldots, m$.

If $L_{1} \Lambda_{1}+L_{2} \Lambda_{2}<1$ then the problem (1.3) has a unique solution on J.

Proof We define a closed ball $B_{r}$ by $B_{r}=\{x \in P C$; $\|x\| \leq r\}$ where $r \geq\left(M_{1} \Lambda_{1}+M_{2} \Lambda_{2}\right)(1-$ $\left.L_{1} \Lambda_{1}-L_{2} \Lambda_{2}\right)^{-1}$, where $M_{1}=\sup _{t \in J}|f(t, 0)|$ and $M_{2}=\max \left\{\left|\varphi_{i}(0)\right|, i=1,2, \ldots, m\right\}$.

We will show that $\mathcal{K}: B_{r} \rightarrow B_{r}$. For any $x \in B_{r}$, we have

$$
\begin{aligned}
& |\mathcal{K} x(t)| \leq \mathcal{J}_{t_{k}}^{p_{k}}|f(t, x(t))|+\sum_{i=0}^{k-1}\left(\mathcal{J}_{t_{i}}^{p_{i}}\left|f\left(t_{i+1}, x\left(t_{i+1}\right)\right)\right|+\left|\varphi_{i+1}\left(x\left(t_{i+1}\right)\right)\right|\right) \\
& +\frac{1}{|\Phi|}\left[\sum_{i=0}^{m}\left|\gamma_{i}\right| \mathcal{J}_{t_{i}}^{q_{i}+p_{i}}\left|f\left(t_{i+1}, x\left(t_{i+1}\right)\right)\right|+|\beta| \mathcal{J}_{t_{m}}^{p_{m}}|f(T, x(T))|\right. \\
& +|\beta| \sum_{i=0}^{m-1}\left(\mathcal{J}_{t_{i}}^{p_{i}}\left|f\left(t_{i+1}, x\left(t_{i+1}\right)\right)\right|+\left|\varphi_{i+1}\left(x\left(t_{i+1}\right)\right)\right|\right) \\
& \left.+\sum_{i=1}^{m}\left(\frac{\left|\gamma_{i}\right|\left(\log \left(t_{i+1} / t_{i}\right)\right)^{q_{i}}}{\Gamma\left(q_{i}+1\right)}\right)\left(\sum_{j=0}^{i-1}\left(\mathcal{J}_{t_{j}}^{p_{j}}\left|f\left(t_{j+1}, x\left(t_{j+1}\right)\right)\right|+\left|\varphi_{j+1}\left(x\left(t_{j+1}\right)\right)\right|\right)\right)\right] \\
& \leq \mathcal{J}_{t_{k}}^{p_{k}}(|f(t, x(t))-f(t, 0)|+|f(t, 0)|) \\
& +\sum_{i=0}^{k-1}\left(\mathcal{J}_{t_{i}}^{p_{i}}\left(\left|f\left(t_{i+1}, x\left(t_{i+1}\right)\right)-f\left(t_{i+1}, 0\right)\right|+\left|f\left(t_{i+1}, 0\right)\right|\right)\right. \\
& \left.+\left|\varphi_{i+1}\left(x\left(t_{i+1}\right)\right)-\varphi_{i+1}(0)\right|+\left|\varphi_{i+1}(0)\right|\right) \\
& +\frac{1}{|\Phi|}\left[\sum_{i=0}^{m}\left|\gamma_{i}\right| \mathcal{J}_{t_{i}}^{q_{i}+p_{i}}\left(\left|f\left(t_{i+1}, x\left(t_{i+1}\right)\right)-f\left(t_{i+1}, 0\right)\right|+\left|f\left(t_{i+1}, 0\right)\right|\right)\right. \\
& +|\beta| \mathcal{J}_{t_{m}}^{p_{m}}(|f(T, x(T))-f(T, 0)|+|f(T, 0)|) \\
& +|\beta| \sum_{i=0}^{m-1}\left(\mathcal{J}_{t_{i}}^{p_{i}}\left(\left|f\left(t_{i+1}, x\left(t_{i+1}\right)\right)-f\left(t_{i+1}, 0\right)\right|+\left|f\left(t_{i+1}, 0\right)\right|\right)\right.
\end{aligned}
$$




$$
\begin{aligned}
& \left.+\left|\varphi_{i+1}\left(x\left(t_{i+1}\right)\right)-\varphi_{i+1}(0)\right|+\left|\varphi_{i+1}(0)\right|\right) \\
& +\sum_{i=1}^{m}\left(\frac{\left|\gamma_{i}\right|\left(\log \left(t_{i+1} / t_{i}\right)\right)^{q_{i}}}{\Gamma\left(q_{i}+1\right)}\right)\left(\sum _ { j = 0 } ^ { i - 1 } \left(\mathcal { J } _ { t _ { j } } ^ { p _ { j } } \left(\left|f\left(t_{j+1}, x\left(t_{j+1}\right)\right)-f\left(t_{j+1}, 0\right)\right|\right.\right.\right. \\
& \left.\left.\left.\left.+\left|f\left(t_{j+1}, 0\right)\right|\right)+\left|\varphi_{j+1}\left(x\left(t_{j+1}\right)\right)-\varphi_{j+1}(0)\right|+\left|\varphi_{j+1}(0)\right|\right)\right)\right] \\
& \leq\left(L_{1} r+M\right) \frac{\left(\log \left(t / t_{k}\right)\right)^{p_{k}}}{\Gamma\left(p_{k}+1\right)}+\sum_{i=0}^{k-1}\left\{\left(L_{1} r+M_{1}\right) \frac{\left(\log \left(t_{i+1} / t_{i}\right)\right)^{p_{i}}}{\Gamma\left(p_{i}+1\right)}+\left(L_{2} r+M_{2}\right)\right\} \\
& +\frac{1}{|\Phi|}\left[\sum_{i=0}^{m}\left|\gamma_{i}\right|\left(L_{1} r+M_{1}\right) \frac{\left(\log \left(t_{i+1} / t_{i}\right)\right)^{q_{i}+p_{i}}}{\Gamma\left(q_{i}+p_{i}+1\right)}+|\beta|\left(L_{1} r+M_{1}\right) \frac{\left(\log \left(T / t_{m}\right)\right)^{p_{m}}}{\Gamma\left(p_{m}+1\right)}\right. \\
& +|\beta| \sum_{i=0}^{m-1}\left\{\left(L_{1} r+M_{1}\right) \frac{\left(\log \left(t_{i+1} / t_{i}\right)\right)^{p_{i}}}{\Gamma\left(p_{i}+1\right)}+\left(L_{2} r+M_{2}\right)\right\} \\
& \left.+\sum_{i=1}^{m}\left\{\left(\frac{\left|\gamma_{i}\right|\left(\log \left(t_{i+1} / t_{i}\right)\right)^{q_{i}}}{\Gamma\left(q_{i}+1\right)}\right)\left(\sum_{j=0}^{i-1}\left(L_{1} r+M_{1}\right) \frac{\left(\log \left(t_{j+1} / t_{j}\right)\right)^{p_{j}}}{\Gamma\left(p_{j}+1\right)}+\left(L_{2} r+M_{2}\right)\right)\right\}\right] \\
& \leq\left(L_{1} \Lambda_{1}+L_{2} \Lambda_{2}\right) r+\left(M_{1} \Lambda_{1}+M_{2} \Lambda_{2}\right) \leq r .
\end{aligned}
$$

Then $\mathcal{K} B_{r} \subseteq B_{r}$. Next we will show that $\mathcal{K}$ is a contraction mapping. For $x, y \in B_{r}$, we get

$$
\begin{aligned}
\mid \mathcal{K} x & -\mathcal{K}_{y} \mid \\
\leq & \mathcal{J}_{t_{k}}^{p_{k}}|f(t, x(t))-f(t, y(t))|+\sum_{i=0}^{k-1}\left(\mathcal{J}_{t_{i}}^{p_{i}}\left|f\left(t_{i+1}, x\left(t_{i+1}\right)\right)-f\left(t_{i+1}, y\left(x_{i+1}\right)\right)\right|\right. \\
& \left.+\left|\varphi_{i+1}\left(x\left(t_{i+1}\right)\right)-\varphi_{i+1}\left(y\left(t_{i+1}\right)\right)\right|\right) \\
& +\frac{1}{|\Phi|}\left[\sum_{i=0}^{m}\left|\gamma_{i}\right| \mathcal{J}_{t_{i}}^{q_{i}+p_{i}}\left|f\left(t_{i+1}, x\left(t_{i+1}\right)\right)-f\left(t_{i+1}, y\left(t_{i+1}\right)\right)\right|\right. \\
& +|\beta| \mathcal{J}_{t_{m}}^{p_{m}}|f(T, x(T))-f(T, y(T))| \\
& +|\beta| \sum_{i=0}^{m-1}\left(\mathcal{J}_{t_{i}}^{p_{i}}\left|f\left(t_{i+1}, x\left(t_{i+1}\right)\right)-f\left(t_{i+1}, y\left(t_{i+1}\right)\right)\right|+\left|\varphi_{i+1}\left(x\left(t_{i+1}\right)\right)-\varphi_{i+1}\left(y\left(t_{i+1}\right)\right)\right|\right) \\
& +\sum_{i=1}^{m}\left(\frac{\left|\gamma_{i}\right|\left(\log \left(t_{i+1} / t_{i}\right)\right)^{q_{i}}}{\Gamma\left(q_{i}+1\right)}\right)\left(\sum _ { j = 0 } ^ { i - 1 } \left(\mathcal{J}_{t_{j}}^{p_{j}}\left|f\left(t_{j+1}, x\left(t_{j+1}\right)\right)-f\left(t_{j+1}, y\left(t_{j+1}\right)\right)\right|\right.\right. \\
& \left.\left.\left.+\left|\varphi_{j+1}\left(x\left(t_{j+1}\right)\right)-\varphi_{j+1}\left(y\left(t_{j+1}\right)\right)\right|\right)\right)\right] \\
\leq & \left(L_{1} \Lambda_{1}+L_{2} \Lambda_{2}\right)\|x-y\| .
\end{aligned}
$$

Since $\left(L_{1} \Lambda_{1}+L_{2} \Lambda_{2}\right)<1$, the operator $\mathcal{K}$ is contractive. Hence $\mathcal{K}$ has a unique fixed point on $B_{r}$. Therefore the problem (1.3) has a unique solution on $J$.

Theorem 3.2 Let $f$ and $\varphi_{k}, k=1,2, \ldots, m$, be continuous functions. Assume that there are two positive real numbers $N_{1}$ and $N_{2}$ such that: 
$\left(\mathrm{H}_{3}\right)|f(t, x)| \leq N_{1}$ and $\left|\varphi_{k}(x)\right| \leq N_{2}$, for $t \in J, x \in \mathbb{R}$ and $k=1,2, \ldots, m$.

Then the problem (1.3) has at least one solution on J.

Proof Define a ball $B_{\omega}=\{x \in P C ;\|x\|<\omega\}$. The proof is divided into 3 steps.

Step 1 . We will show that $\mathcal{K}$ is continuous. To prove this, we let $\left\{x_{n}\right\}$ be a sequence in $P C$ such that $x_{n} \rightarrow x$ as $n \rightarrow \infty$. Then we have

$$
\begin{aligned}
\mid \mathcal{K} x_{n}( & t)-\mathcal{K} x(t) \mid \\
\leq & \mathcal{J}_{t_{k}}^{p_{k}}\left|f\left(t, x_{n}(t)\right)-f(t, x(t))\right| \\
& +\sum_{i=0}^{k-1}\left(\mathcal{J}_{t_{i}}^{p_{i}}\left|f\left(t_{i+1}, x_{n}\left(t_{i+1}\right)\right)-f\left(t_{i+1}, x\left(t_{i+1}\right)\right)\right|+\left|\varphi_{i+1}\left(x_{n}\left(t_{i+1}\right)\right)-\varphi_{i+1}\left(x\left(t_{i+1}\right)\right)\right|\right) \\
& +\frac{1}{|\Phi|}\left[\sum_{i=0}^{m}\left|\gamma_{i}\right| \mathcal{J}_{t_{i}}^{q_{i}+p_{i}}\left|f\left(t_{i+1}, x_{n}\left(t_{i+1}\right)\right)-f\left(t_{i+1}, x\left(t_{i+1}\right)\right)\right|\right. \\
& +|\beta| \mathcal{J}_{t_{m}}^{p_{m}}\left|f\left(T, x_{n}(T)\right)-f(T, x(T))\right| \\
& +|\beta| \sum_{i=0}^{m-1}\left(\mathcal{J}_{t_{i}}^{p_{i}}\left|f\left(t_{i+1}, x_{n}\left(t_{i+1}\right)\right)-f\left(t_{i+1}, x\left(t_{i+1}\right)\right)\right|\right. \\
& \left.+\left|\varphi_{i+1}\left(x_{n}\left(t_{i+1}\right)\right)-\varphi_{i+1}\left(x\left(t_{i+1}\right)\right)\right|\right) \\
& +\sum_{i=1}^{m}\left(\frac{\left|\gamma_{i}\right|\left(\log \left(t_{i+1} / t_{i}\right)\right)^{q_{i}}}{\Gamma\left(q_{i}+1\right)}\right)\left(\sum _ { j = 0 } ^ { i - 1 } \left(\mathcal{J}_{t_{j}}^{p_{j}}\left|f\left(t_{j+1}, x_{n}\left(t_{j+1}\right)\right)-f\left(t_{j+1}, x\left(t_{j+1}\right)\right)\right|\right.\right. \\
& \left.\left.\left.+\left|\varphi_{j+1}\left(x_{n}\left(t_{j+1}\right)\right)-\varphi_{j+1}\left(x\left(t_{j+1}\right)\right)\right|\right)\right)\right]
\end{aligned}
$$

Using the continuity of $f$ and $\varphi_{k}$ for $k=1,2, \ldots, m$, we have $\left|f\left(t, x_{n}\right)-f(t, x)\right|$ and $\mid \varphi_{k}\left(x_{n}\right)-$ $\varphi_{k}(x) \mid$ vanish as $n \rightarrow \infty$. Therefore $\left\|\mathcal{K} x_{n}-\mathcal{K} x\right\| \rightarrow 0$, which yields the continuity of the operator $\mathcal{K}$.

Step 2 . $\mathcal{K}$ maps a bounded set into a bounded set. For each $x \in \bar{B}_{\omega}$, we have

$$
\begin{aligned}
|\mathcal{K} x| \leq & \mathcal{J}_{t_{k}}^{p_{k}}|f(t, x(t))|+\sum_{i=0}^{k-1}\left(\mathcal{J}_{t_{i}}^{p_{i}}\left|f\left(t_{i+1}, x\left(t_{i+1}\right)\right)\right|+\left|\varphi_{i+1}\left(x\left(t_{i+1}\right)\right)\right|\right) \\
& +\frac{1}{|\Phi|}\left[\sum_{i=0}^{m}\left|\gamma_{i}\right| \mathcal{J}_{t_{i}}^{q_{i}+p_{i}}\left|f\left(t_{i+1}, x\left(t_{i+1}\right)\right)\right|+|\beta| \mathcal{J}_{t_{m}}^{p_{m}}|f(T, x(T))|\right. \\
& +|\beta| \sum_{i=0}^{m-1}\left(\mathcal{J}_{t_{i}}^{p_{i}}\left|f\left(t_{i+1}, x\left(t_{i+1}\right)\right)\right|+\left|\varphi_{i+1}\left(x\left(t_{i+1}\right)\right)\right|\right) \\
& \left.+\sum_{i=1}^{m}\left(\frac{\left|\gamma_{i}\right|\left(\log \left(t_{i+1} / t_{i}\right)\right)^{q_{i}}}{\Gamma\left(q_{i}+1\right)}\right)\left(\sum_{j=0}^{i-1}\left(\mathcal{J}_{t_{j}}^{p_{j}}\left|f\left(t_{j+1}, x\left(t_{j+1}\right)\right)\right|+\left|\varphi_{j+1}\left(x\left(t_{j+1}\right)\right)\right|\right)\right)\right] \\
\leq & \Lambda_{1} N_{1}+\Lambda_{2} N_{2},
\end{aligned}
$$

which yields the boundedness of $\mathcal{K} \bar{B}_{\omega}$. 
Step 3. $\mathcal{K}$ maps a bounded set into an equicontinuous set. Let $\tau_{1}, \tau_{2} \in\left(t_{k}, t_{k+1}\right)$, for each $k=0,1,2, \ldots, m$, we have

$$
\left|\mathcal{K} x\left(\tau_{1}\right)-\mathcal{K} x\left(\tau_{2}\right)\right| \leq \mathcal{J}_{t_{k}}^{p_{k}}\left|f\left(\tau_{1}, x\left(\tau_{1}\right)\right)-f\left(\tau_{2}, x\left(\tau_{2}\right)\right)\right|
$$

The continuity of $x$ and $f$ implies that $\mathcal{K} x\left(\tau_{1}\right) \rightarrow \mathcal{K} x\left(\tau_{2}\right)$ as $\tau_{1} \rightarrow \tau_{2}$. Consequently $\mathcal{K}$ is completely continuous by applying the Azelá-Ascoli theorem.

Let $V=\left\{x \in B_{\omega} ; \mu \mathcal{K} x=x\right.$ for $\left.\mu \in(0,1)\right\}$. For all $x \in V, x=\mu \mathcal{K} x$, we have

$$
|x| \leq \mu|\mathcal{K} x| \leq \Lambda_{1} N_{1}+\Lambda_{2} N_{2}
$$

Hence $V$ is bounded. By Theorem 2.3, the problem (1.3) has at least one solution on $J$.

Theorem 3.3 Assume that

$\left(\mathrm{H}_{4}\right) \lim _{x \rightarrow 0} \frac{f(t, x)}{x}=0$ and $\lim _{x \rightarrow 0} \frac{\varphi_{k}(x)}{x}=0$ for $k=1,2, \ldots, m$.

Then the problem (1.3) has at least one solution on J.

Proof From $\left(\mathrm{H}_{4}\right)$, choosing $\epsilon=1 /\left(\Lambda_{1}+\Lambda_{2}\right)$, there exist constants $\delta_{1}, \delta_{2} \in \mathbb{R}^{+}$such that

$$
|f(t, x)|<\epsilon|x| \text { where }|x|<\delta_{1} \text { and }|\varphi(x)|<\epsilon|x| \quad \text { where }|x|<\delta_{2} \text {. }
$$

Now, we define an open ball $\Omega=\left\{u \in P C ;\|u\|<\min \left\{\delta_{1}, \delta_{2}\right\}\right\}$. By Theorem 3.2, the operator $\mathcal{K}: \bar{\Omega} \rightarrow P C$ is completely continuous. For any $x \in \partial \Omega$, we have

$$
\begin{aligned}
|\mathcal{K} x| \leq & \mathcal{J}_{t_{k}}^{p_{k}}|f(t, x(t))|+\sum_{i=0}^{k-1}\left(\mathcal{J}_{t_{i}}^{p_{i}}\left|f\left(t_{i+1}, x\left(t_{i+1}\right)\right)\right|+\left|\varphi_{i+1}\left(x\left(t_{i+1}\right)\right)\right|\right) \\
& +\frac{1}{|\Phi|}\left[\sum_{i=0}^{m}\left|\gamma_{i}\right| \mathcal{J}_{t_{i}}^{q_{i}+p_{i}}\left|f\left(t_{i+1}, x\left(t_{i+1}\right)\right)\right|+|\beta| \mathcal{J}_{t_{m}}^{p_{m}}|f(T, x(T))|\right. \\
& +|\beta| \sum_{i=0}^{m-1}\left(\mathcal{J}_{t_{i}}^{p_{i}}\left|f\left(t_{i+1}, x\left(t_{i+1}\right)\right)\right|+\left|\varphi_{i+1}\left(x\left(t_{i+1}\right)\right)\right|\right) \\
& \left.+\sum_{i=1}^{m}\left(\frac{\left|\gamma_{i}\right|\left(\log \left(t_{i+1} / t_{i}\right)\right)^{q_{i}}}{\Gamma\left(q_{i}+1\right)}\right)\left(\sum_{j=0}^{i-1}\left(\mathcal{J}_{t_{j}}^{p_{j}}\left|f\left(t_{j+1}, x\left(t_{j+1}\right)\right)\right|+\left|\varphi_{j+1}\left(x\left(t_{j+1}\right)\right)\right|\right)\right)\right] \\
\leq & \left(\Lambda_{1} \epsilon+\Lambda_{2} \epsilon\right)\|x\|=\|x\| .
\end{aligned}
$$

It follows from Theorem 2.1, case (ii), that the problem (1.3) has at least one solution on $J$

Theorem 3.4 Let $f$ and $\varphi_{k}$ for $k=1,2, \ldots, m$, be continuous functions and satisfy the following inequalities:

$\left(\mathrm{H}_{5}\right) \quad|f(t, x)| \leq a|x|+b, \forall(t, x) \in J \times \mathbb{R}$ and $\left|\varphi_{k}(x)\right| \leq c|x|+d, \forall x \in \mathbb{R}, k=1, \ldots, m$, where constants $a, c>0$ and $b, d \geq 0$.

Then the problem (1.3) has at least one solution on J. 
Proof Define a unit ball as $\mathcal{O}=\{x \in P C ;\|x\|<1\}$. It is straightforward to show that the operator $\mathcal{K}: \overline{\mathcal{O}} \rightarrow P C$ is completely continuous. Suppose that there is $x^{*} \in \partial \mathcal{O}$. Then we choose $\lambda=(a+c) \Lambda_{1}+(b+d) \Lambda_{2}+1$ such that $\mathcal{K} x^{*}=\lambda x^{*}$. By taking the norm in both sides of $\left\|\mathcal{K} x^{*}\right\|=\left\|\lambda x^{*}\right\|$, we obtain $\|\mathcal{K}\|\left\|x^{*}\right\| \geq \lambda\left\|x^{*}\right\|$. Then we have

$$
\begin{aligned}
\|\mathcal{K}\|= & \sup _{\|x\|=1}|\mathcal{K} x| \\
= & \sup _{\|x\|=1}\left\{\mathcal{J}_{t_{k}}^{p_{k}}|f(t, x(t))|+\sum_{i=0}^{k-1}\left(\mathcal{J}_{t_{i}}^{p_{i}}\left|f\left(t_{i+1}, x\left(t_{i+1}\right)\right)\right|+\left|\varphi_{i+1}\left(x\left(t_{i+1}\right)\right)\right|\right)\right. \\
& +\frac{1}{|\Phi|}\left[\sum_{i=0}^{m}\left|\gamma_{i}\right| \mathcal{J}_{t_{i}}^{q_{i}+p_{i}}\left|f\left(t_{i+1}, x\left(t_{i+1}\right)\right)\right|+|\beta| \mathcal{J}_{t_{m}}^{p_{m}}|f(T, x(T))|\right. \\
& +|\beta| \sum_{i=0}^{m-1}\left(\mathcal{J}_{t_{i}}^{p_{i}}\left|f\left(t_{i+1}, x\left(t_{i+1}\right)\right)\right|+\left|\varphi_{i+1}\left(x\left(t_{i+1}\right)\right)\right|\right) \\
& \left.\left.+\sum_{i=1}^{m}\left(\frac{\left|\gamma_{i}\right|\left(\log \left(t_{i+1} / t_{i}\right)\right)^{q_{i}}}{\Gamma\left(q_{i}+1\right)}\right)\left(\sum_{j=0}^{i-1}\left(\mathcal{J}_{t_{j}}^{p_{j}}\left|f\left(t_{j+1}, x\left(t_{j+1}\right)\right)\right|+\left|\varphi_{j+1}\left(x\left(t_{j+1}\right)\right)\right|\right)\right)\right]\right\} \\
\leq & (a+c) \Lambda_{1}+(b+d) \Lambda_{2}=\lambda-1,
\end{aligned}
$$

which contradicts $\|\mathcal{K}\| \geq \lambda$. Hence the assumptions of Theorem 2.2 hold. Therefore the problem (1.3) has at least one solution on $J$.

\section{Examples}

In this section, we present four examples to illustrate our results.

Example 4.1 Consider the boundary value problem for an impulsive multi-order Hadamard fractional differential equation of the form

$$
\left\{\begin{array}{l}
{ }^{C} \mathcal{D}_{t_{k}}^{\left(\frac{k+1}{k+2}\right)} x(t)=\frac{10-t^{2}}{8\left(t^{2}+24\right)}\left(\frac{(|x(t)|+2)^{2}}{|x(t)|+3}\right), \quad t \in\left[1, \frac{8 e+1}{9}\right] \backslash\left\{t_{k}\right\}, \\
\Delta x\left(t_{k}\right)=\frac{\sin \left|x\left(t_{k}\right)\right|}{5(11-k)}, \quad t_{k}=\frac{k e+1}{k+1}, k=1,2, \ldots, 7, \\
\frac{3}{2} x(1)+\frac{4}{5} x\left(\frac{8 e+1}{9}\right)=\sum_{i=0}^{7}\left(1-e^{-i}\right) \mathcal{J}_{t_{i}}^{\left(\frac{i^{2}+5 i+2}{i^{2}+4 i+3}\right)} x\left(t_{i+1}\right) .
\end{array}\right.
$$

Here $\alpha=3 / 2, \beta=4 / 5, m=7, p_{k}=(k+1) /(k+2), \gamma_{k}=1-e^{-k}, q_{k}=\left(k^{2}+5 k+2\right) /\left(k^{2}+4 k+3\right)$ for $k=0,1, \ldots, 7$. From the information, we find that $\Phi \approx 2.0961081, \Lambda_{1} \approx 3.280445$, and $\Lambda_{2} \approx 13.552466$. The functions $f$ and $\varphi_{k}$ are given by

$$
f(t, x)=\frac{10-t^{2}}{8\left(t^{2}+24\right)}\left(\frac{(|x|+2)^{2}}{|x|+3}\right), \quad \varphi_{k}(x)=\frac{\sin |x|}{5(11-k)},
$$

which satisfy

$$
|f(t, x)-f(t, y)| \leq \frac{2}{25}|x-y| \quad \text { and } \quad\left|\varphi_{k}(x)-\varphi_{k}(y)\right| \leq \frac{1}{20}|x-y|, \quad \forall k=1,2, \ldots, 7 .
$$

Then we get $L_{1}=2 / 25$ and $L_{2}=1 / 20$, which implies $L_{1} \Lambda_{1}+L_{2} \Lambda_{2} \approx 0.940059<1$. Therefore the problem (4.1) has a unique solution on $[1,(8 e+1) / 9]$ due to Theorem 3.1 . 
Example 4.2 Consider the boundary value problem for an impulsive multi-order Hadamard fractional differential equation of the form

$$
\left\{\begin{array}{l}
{ }^{C} \mathcal{D}_{t_{k}}^{\log \left(\sum_{i=0}^{k+1}(1 /(i+1) !)\right)} x(t)=\frac{\left(2-e^{-t}\right) \log (|x(t)|+1)}{|x(t)|+2}-2, \quad t \in\left[\frac{\pi}{2}, 2 \pi\right] \backslash\left\{t_{k}\right\}, \\
\Delta x\left(t_{k}\right)=e^{-\frac{k}{4}} \cos \left(k x\left(t_{k}\right)\right)+e^{\frac{k}{4}} \sin \left(k x\left(t_{k}\right)\right), \quad t_{k}=2^{\frac{2 k-9}{9}} \pi, k=1,2, \ldots, 8, \\
-e^{-\frac{\pi}{2}} x\left(\frac{\pi}{2}\right)+e^{-2 \pi} x(2 \pi)=\sum_{i=0}^{8}(-2)^{i}\left(i^{2}+1\right) \mathcal{J}_{t_{i}}^{\left(\frac{\mid 5 i-4}{i+1}\right)} x\left(t_{i+1}\right) .
\end{array}\right.
$$

Here $\alpha=-e^{-\pi / 2}, \beta=e^{-2 \pi}, m=8, p_{k}=\log \left(\sum_{i=0}^{k+1}(1 /(i+1) !)\right), \gamma_{k}=(-2)^{k}\left(k^{2}+1\right), q_{k}=\mid 5 k-$ $4 \mid /(k+1)$ for $k=0,1, \ldots, 8$. We find that $\Phi \approx-1.422922 \neq 0$. The functions $f(t, x)=(2-$ $\left.e^{-t}\right) \log (|x|+1) /(|x|+2)-2$ and $\varphi_{k}(x)=e^{-\frac{k}{4}} \cos (k x)+e^{\frac{k}{4}} \sin (k x)$ are bounded as

$$
|f(t, x)| \leq 4 \quad \text { and } \quad\left|\varphi_{k}(x)\right| \leq \sqrt{e^{-4}+e^{4}} .
$$

Hence the assumption $\left(\mathrm{H}_{3}\right)$ of Theorem 3.2 holds. Therefore the problem (4.2) has at least one solution on $[\pi / 2,2 \pi]$.

Example 4.3 Consider the boundary value problem for an impulsive multi-order Hadamard fractional differential equation of the form

$$
\left\{\begin{array}{l}
{ }^{C} \mathcal{D}_{t_{k}}^{\left(\frac{2 k+2}{k^{2}+2 k+2}\right)} x(t)=\frac{e^{t x x(t)}(\sin x(t)-x(t))}{2 t+1}, \quad t \in\left[\frac{4}{3}, 3\right] \backslash\left\{t_{k}\right\} \\
\Delta x\left(t_{k}\right)=\frac{k x^{3}\left(t_{k}\right)}{\log \left(\left|x\left(t_{k}\right)\right|+2\right)}, \quad t_{k}=\frac{k+8}{6}, k=1,2, \ldots, 9 \\
\sqrt{3} x\left(\frac{4}{3}\right)+\frac{3}{5} x(3)=\sum_{i=0}^{9}\left(\frac{i^{2}+1}{i^{2}+2}\right) \mathcal{J}_{t_{i}}^{\arctan i} x\left(t_{i+1}\right) .
\end{array}\right.
$$

Here $\alpha=\sqrt{3}, \beta=3 / 5, m=9, p_{k}=2(k+1) /\left(k^{2}+1\right), \gamma_{k}=\left(k^{2}+1\right) /\left(k^{2}+2\right), q_{k}=\arctan (k)$ for $k=0,1, \ldots, 9$. We find that $\Phi \approx 2.003684 \neq 0$. The functions $f(t, x)=e^{t x}(\sin x-x) /(2 t+1)$ and $\varphi_{k}(x)=k x^{3} / \log (|x|+2)$ satisfy

$$
\lim _{x \rightarrow 0} \frac{f(t, x)}{x}=\lim _{x \rightarrow 0} \frac{e^{x t}}{2 t+1}\left(\frac{\sin x}{x}-1\right)=0
$$

and

$$
\lim _{x \rightarrow 0} \frac{\varphi_{k}(x)}{x}=\lim _{x \rightarrow 0} \frac{k x^{2}}{\log (|x|+2)}=0, \quad \forall k=1,2, \ldots, 9 .
$$

Thus the condition $\left(\mathrm{H}_{4}\right)$ of Theorem 3.3 holds. Therefore, we conclude that the problem (4.3) has at least one solution on $[4 / 3,3]$.

Example 4.4 Consider the boundary value problem for an impulsive multi-order Hadamard fractional differential equation of the form

$$
\left\{\begin{array}{l}
{ }^{C} \mathcal{D}_{t_{k}}^{\sqrt{1-\sin ^{2}(k+1)}} x(t)=e^{\frac{2 t}{3}} \sin x(t)+t x(t) \cos x(t)+2, \quad t \in\left[\frac{3}{2}, 3\right] \backslash\left\{t_{k}\right\}, \\
\Delta x\left(t_{k}\right)=k x\left(t_{k}\right)-\log \left(\left|x\left(t_{k}\right)\right|+\frac{3}{5}\right), \quad t_{k}=3 \cdot 2^{\frac{k-11}{11}}, k=1,2, \ldots, 10, \\
\frac{4}{3} x\left(\frac{3}{2}\right)-\frac{3}{4} x(3)=\sum_{i=0}^{10} \frac{(-1)^{i}}{i+1} \mathcal{J}_{t_{i}}^{\left(\frac{3 k+2}{2 k+3}\right)} x\left(t_{i+1}\right) .
\end{array}\right.
$$


Here $\alpha=4 / 3, \beta=-3 / 4, m=10, p_{k}=\sqrt{1-\sin ^{2}(k+1)}, \gamma_{k}=(-1)^{k} /(k+1), q_{k}=(3 k+$ $2) /(2 k+3)$, for $k=0,1, \ldots, 10$. We find that $\Phi \approx 0.605503 \neq 0$. The functions $f(t, x)=$ $e^{\frac{2 t}{3}} \sin x+t x \cos x+2$ and $\varphi(x)=k x-\log (|x|+(3 / 5))$ satisfy the inequalities

$$
|f(t, x)| \leq t|x|+\left(2+e^{\frac{2 t}{3}}\right) \leq 3|x|+\left(2+e^{2}\right)
$$

and

$$
\left|\varphi_{k}(x)\right| \leq|x|(k+1)+\frac{3}{5} \leq 11|x|+\frac{3}{5}
$$

Therefore $\left(H_{5}\right)$ holds. According to Theorem 3.4, the problem (4.4) has at least one solution on $[3 / 2,3]$.

\section{Competing interests}

The authors declare that they have no competing interests.

\section{Authors' contributions}

All authors contributed equally in this article. They read and approved the final manuscript.

\section{Author details}

${ }^{1}$ Nonlinear Dynamic Analysis Research Center, Department of Mathematics, Faculty of Applied Science, King Mongkut's University of Technology North Bangkok, Bangkok, 10800, Thailand. ${ }^{2}$ Department of Mathematics, Faculty of Science, Chiang Mai University, Chiang Mai, 50200, Thailand. ${ }^{3}$ Department of Mathematics, University of loannina, loannina, 451 10, Greece. ${ }^{4}$ Nonlinear Analysis and Applied Mathematics (NAAM)-Research Group, Department of Mathematics, Faculty of Science, King Abdulaziz University, P.O. Box 80203, Jeddah, 21589, Saudi Arabia.

\section{Acknowledgements}

This paper was supported by the Thailand Research Fund under the project RTA5780007.

Received: 21 May 2015 Accepted: 11 August 2015 Published online: 28 August 2015

\section{References}

1. Kilbas, AA, Srivastava, HM, Trujillo, JJ: Theory and Applications of Fractional Differential Equations. North-Holland Mathematics Studies, vol. 204. Elsevier Science B.V., Amsterdam (2006)

2. Miller, KS, Ross, B: An Introduction to the Fractional Calculus and Fractional Differential Equations. Wiley, New York (1993)

3. Podlubny, I: Fractional Differential Equations. Academic Press, San Diego (1999)

4. Ahmad, B, Ntouyas, SK, Alsaedi, A: An existence result for fractional differential inclusions with nonlinear integral boundary conditions. J. Inequal. Appl. 2013, 296 (2013)

5. Nyamoradi, N, Baleanu, D, Agarwal, RP: On a multipoint boundary value problem for a fractional order differential inclusion on an infinite interval. Adv. Math. Phys. 2013, Article ID 823961 (2013)

6. Ntouyas, SK, Tariboon, J, Sudsutad, W: Boundary value problems for Riemann-Liouville fractional differential inclusions with nonlocal Hadamard fractional integral conditions. Meditter. J. Math. (2015). doi:10.1007/s00009-015-0543-1

7. Alsaedi, A, Ntouyas, SK, Agarwal, RP, Ahmad, B: On Caputo type sequential fractional differential equations with nonlocal integral boundary conditions. Adv. Differ. Equ. 2015, 33 (2015)

8. Yukunthorn, W, Ntouyas, SK, Tariboon, J: Nonlinear fractional Caputo-Langevin equation with nonlocal Riemann-Liouville fractional integral conditions. Adv. Differ. Equ. 2014, 315 (2014)

9. Tariboon, J, Ntouyas, SK, Sudsutad, W: Nonlocal Hadamard fractional integral conditions for nonlinear Riemann-Liouville fractional differential equations. Bound. Value Probl. 2014, 253 (2014)

10. Hadamard, J: Essai sur l'étude des fonctions données par leur développement de Taylor. J. Mat. Pure Appl. Ser. 8 101-186 (1892)

11. Butzer, PL, Kilbas, AA, Trujillo, JJ: Compositions of Hadamard-type fractional integration operators and the semigroup property. J. Math. Anal. Appl. 269, 387-400 (2002)

12. Butzer, PL, Kilbas, AA, Trujillo, J: Fractional calculus in the Mellin setting and Hadamard-type fractional integrals. J. Math. Anal. Appl. 269, 1-27 (2002)

13. Butzer, PL, Kilbas, AA, Trujillo, JJ: Mellin transform analysis and integration by parts for Hadamard-type fractional integrals. J. Math. Anal. Appl. 270, 1-15 (2002)

14. Kilbas, AA: Hadamard-type fractional calculus. J. Korean Math. Soc. 38, 1191-1204 (2001)

15. Kilbas, AA, Trujillo, JJ: Hadamard-type integrals as G-transforms. Integral Transforms Spec. Funct. 14, 413-427 (2003)

16. Agarwal, RP, Ahmad, B: Existence of solutions for impulsive anti-periodic boundary value problems of fractional semilinear evolution equations. Dyn. Contin. Discrete Impuls. Syst., Ser. A Math. Anal. 18, 535-544 (2011)

17. Samoilenko, AM, Perestyuk, NA: Impulsive Differential Equations. World Scientific, Singapore (1995)

18. Benchohra, M, Henderson, J, Ntouyas, SK: Impulsive Differential Equations and Inclusions. Hindawi Publishing, New York (2006) 
19. Ahmad, B, Wang, G: A study of an impulsive four-point nonlocal boundary value problem of nonlinear fractional differential equations. Comput. Math. Appl. 62, 1341-1349 (2011)

20. Zhang, $X$, Huang, $X, L i u, Z$ : The existence and uniqueness of mild solutions for impulsive fractional equations with nonlocal conditions and infinite delay. Nonlinear Anal. Hybrid Syst. 4, 775-781 (2010)

21. Wang, G, Liu, S, Baleanu, D, Zhang, L: A new impulsive multi-orders fractional differential equation involving multipoint fractional integral boundary conditions. Abstr. Appl. Anal. 2014, Article ID 932747 (2014)

22. Wang, J, Zhang, Y: On the concept and existence of solutions for fractional impulsive systems with Hadamard derivatives. Appl. Math. Lett. 39, 85-90 (2015)

23. Jarad, F, Abdeljawad, T, Baleanu, D: Caputo-type modification of the Hadamard fractional derivatives. Adv. Differ. Equ. 2012, 142 (2012)

24. Gambo, YY, Jarad, F, Baleanu, D, Abdeljawad, T: On Caputo modification of the Hadamard fractional derivatives. Adv. Differ. Equ. 2014, 10 (2014)

25. Sun, L, Sun, J, Wang, G: Generalizations of fixed-point theorems of Altman and Rothe types. Abstr. Appl. Anal. 2013, Article ID 639030 (2013)

26. Granas, A, Dugundji, J: Fixed Point Theory. Springer, New York (2003)

\section{Submit your manuscript to a SpringerOpen ${ }^{\circ}$ journal and benefit from:}

- Convenient online submission

Rigorous peer review

- Immediate publication on acceptance

- Open access: articles freely available online

- High visibility within the field

- Retaining the copyright to your article 